\title{
Expression Levels of Human Equilibrative Nucleoside Transporter 1 and Deoxycytidine Kinase Enzyme as Prognostic Factors in Patients with Acute Myeloid Leukemia Treated with Cytarabine
}

\author{
Myrna Candelariaa, ${ }^{a} \quad$ Carmen Corrales-Alfarob ${ }^{b}$ Olga Gutiérrez-Hernández ${ }^{a}$ \\ José Díaz-Chavez ${ }^{c}$ Juan Labardini-Méndez ${ }^{\mathrm{b}}$ Silvia Vidal-Millán ${ }^{a}$ \\ Luis A. Herrera ${ }^{c}$ \\ a Pharmacogenetic Laboratory and b Hematology Department, Instituto Nacional de Cancerología, and \\ 'Unidad de Investigación Biomédica en Cáncer, Instituto de Investigaciones Biomédicas, UNAM/Instituto \\ Nacional de Cancerología, Mexico City, Mexico
}

\section{Key Words}

Pharmacogenetics · Cytarabine response $\cdot$ Nucleoside transporters · Acute myeloid leukemia

\begin{abstract}
Background: Cytarabine (Ara-C) is the primary drug in different treatment schemas for acute myeloid leukemia (AML) and requires the human equilibrative nucleoside transporter (hENT1) to enter cells. The deoxycytidine kinase (dCK) enzyme limits its activation rate. Therefore, decreased expression levels of these genes may influence the response rate to this drug. Methods: AML patients without previous treatment were enrolled. The expression of $h E N T 1$ and $d C K$ genes was analyzed using RT-PCR. Clinical parameters were registered. All patients received Ara- $\mathrm{C}+$ doxorubicin as an induction regimen $(7+3$ schema). Descriptive statistics were used to analyze data. Uni- and multivariate analyses were performed to determine factors that influenced response and survival. Results: Twenty-eight patients were included from January 2011 until December 2012. Median age was 36.5 years. All patients had an adequate performance status ( $43 \%$
\end{abstract}

\section{KARGER}

E-Mail karger@karger.com

www.karger.com/che with ECOG 1 and 57\% with ECOG 2). Cytogenetic risk was considered unfavorable in $54 \%$ of the patients. Complete response was achieved in 53.8\%. Cox regression analysis showed that a higher $h E N T 1$ expression level was the only factor that influenced response and survival. Conclusions: These results highly suggest that the pharmacogenetic analyses of Ara-C influx may be decisive in AML patients.

(c) 2016 S. Karger AG, Basel

\section{Introduction}

Cytarabine (Ara-C) is a cytosine analog that is currently indicated for the treatment of acute myeloid leukemia (AML) [1]. Ara-C is a prodrug that requires cellular influx primarily through the human equilibrative nucleoside transporter ( $h E N T 1$ ) and active phosphorylation within the cell by the deoxycytidine kinase (dCK) enzyme to produce a monophosphorylated product (dCMP), which is bi- and triphosphorylated to act as an antimetabolite. The cytidine deaminase enzyme catalyzes this prodrug. The antineoplasic activity of Ara-C occurs via
(C) 2016 S. Karger AG Basel

0009-3157/16/0616-0313\$39.50/0 
two independent mechanisms: triphosphorylated metabolite (dCTP) inhibits DNA polymerase and then acts as an antimetabolite in DNA synthesis [2, 3].

Cell lines with lower dCK activity are resistant to nucleoside analogs and cells with higher activity are associated with higher sensitivity to these agents $[4,5]$. Similarly, a lineal correlation between mRNA expression levels and enzymatic activity has been demonstrated [6], and decreased $d C K$ expression also lowers activity in Ara-Cresistant cell lines [7]. The expression of hENT1 is also correlated with intracellular Ara-C concentrations and clinical response $[4,5,8]$. The mechanism of primary Ara-C resistance is not completely defined, but inadequate cellular influx, either secondary to the downregulation of $h E N T 1$ expression, reduced dCK activity or the increased activity of the enzymes responsible for Ara-C metabolism, such as $5^{\prime}$-nucleotidase (NT5C2) and cytidine deaminase, may play a role. Therefore, we evaluated the expression levels of the $h E N T 1$ and $d C K$ genes and correlated their expression with response rates and survival in patients with de novo AML.

\section{Patients and Methods}

\section{Patient Samples}

A prospective, pilot, open trial was designed. De novo AML patients, $\geq 15$ years of age, with adequate renal and liver function, who were candidates for treatment with Ara-C $\left(100 \mathrm{mg} / \mathrm{m}^{2} / \mathrm{dose}\right.$ every $12 \mathrm{~h}$ for 7 days $)+$ doxorubicin $\left(60 \mathrm{mg} / \mathrm{m}^{2} /\right.$ day on days $\left.1-3\right)$ in a $7+3$ schema were included. Patients with a poor performance status $($ ECOG $>3$ ) were excluded. Peripheral blood $(15 \mathrm{ml})$ was collected from the untreated patients. Additionally, blood samples from healthy volunteers were collected to compare expression levels of the $h E N T 1$ and $d C K$ genes. RNA was isolated using standard methods, and the $h E N T 1$ and $d C K$ genes were amplified as follows. RNA was extracted using the RNA kit PCR Core Gene Amp ${ }^{\circledR}$ (manufactured for Applied Biosystems by Roche Molecular Systems, Inc., Branchburg, N.J., USA) following the provider's recommendations. The local IRB approved this protocol, which was registered with www.clinicaltrials.gov (Identifier No. NCT01307241). All patients provided signed informed consent.

\section{Real-Time Quantitative PCR}

Each sample was tested in triplicate using quantitative PCR, and mRNA ratios relative to the $18 S$ housekeeping gene were calculated to standardize gene expression levels. The $18 \mathrm{~S}, \mathrm{hENT} 1$ and $d C K$ genes were amplified using the following oligonucleotide primers: 18S: forward: $5^{\prime}$-TCGGAACTGAGGCCATGATT-3' ${ }^{\prime}$, reverse: $5^{\prime}$-CGAACCTCCGACTTTCGTTCT-3'; hENT1: forward: $5^{\prime}$-GCAAAGGAGAGGAGCCAAGA-3', reverse: $5^{\prime}$-CCCAACCAGTCAAAGATATTG-3'; $d C K$ : forward: $5^{\prime}$-CGATCTGTGTATAGTGACAG-3', reverse: $5^{\prime}$-GTTGGTTTTCAGTGTCCTATG-3'. The SYBR Green reaction was performed using the Maxima SYBR Green/ROX qPCR Master Mix $(2 \times)$ reagents kit
Table 1. Baseline clinical characteristics

$\begin{array}{lc}\text { Gender } & \\ \text { Male } & 11(39.3) \\ \text { Female } & 17(60.7) \\ \text { Median age, years (range) } & 36.5(17-75) \\ \text { Performance status } & \\ \text { ECOG 0-1 } & 12(43) \\ \text { ECOG 2 } & 16(57) \\ \text { Cytogenetic risk } & \\ \text { Favorable } & 7(25) \\ \text { Intermediate } & 5(18) \\ \text { Unfavorable } & 15(54) \\ \text { Not evaluable } & 1(3)\end{array}$

Values are expressed as $\mathrm{n}(\%)$, unless otherwise indicated.

(Thermo Scientific, Waltham, Mass., USA) following the manufacturer's recommendations. A reaction optimization was performed for each gene-specific pair of primers before real-time reverse-transcription PCR (RT-qPCR) was performed to confirm the specificity of the amplification signal. The following PCR conditions were used: 1 cycle of $10 \mathrm{~min}$ at $95^{\circ} \mathrm{C}$ and 40 cycles of $30 \mathrm{~s}$ at $95^{\circ} \mathrm{C}, 30 \mathrm{~s}$ at $59^{\circ} \mathrm{C}$ and $30 \mathrm{~s}$ at $72^{\circ} \mathrm{C}$. Changes in fluorescence were recorded as the temperature increased from 65 to $95^{\circ} \mathrm{C}$ at a rate of $0.2^{\circ} \mathrm{C} / \mathrm{s}$ to obtain a DNA melting curve.

Data Analysis Using the $2^{-\triangle \Delta C T}$ Method

Real-time PCR was performed for selected genes on the corresponding cDNA that was synthesized from each sample. Data were analyzed using the equation described by Livak and Schmittgen [9] as follows: amount of target $=2^{-\Delta \Delta C T}$. Validation of the method was performed as reported previously [10]. Data are presented as the mean \pm SD. Statistical evaluation of significant differences was performed using Student's t test. p values $<0.05$ were considered statistically significant. This protocol was approved by the Institutional Ethics Committee and all patients signed informed consent before being included.

Descriptive statistics were used for clinical variables. Expression levels were correlated with response rate and survival. Overall survival (OS) was calculated using the Kaplan-Meier method. Cox regression analyses determined the factors that influenced response and OS.

\section{Results}

Twenty-eight patients were included from January 2011 until December 2012. Their median age was 36.5 years (17-75 years) and $60.7 \%$ were female. The M2 subtype, according to FAB classification, was the most frequent $(39.3 \%)$. All patients had adequate performance status (43\% with ECOG 1 and 66\% with ECOG 2). Cytogenetic risk was assessed as unfavorable (in $72 \%$ ), intermediate (in 18\%) or favorable (in $25 \%$ ) (table 1). 
Fig. 1. Comparison of gene expression between the healthy and leukemic groups.

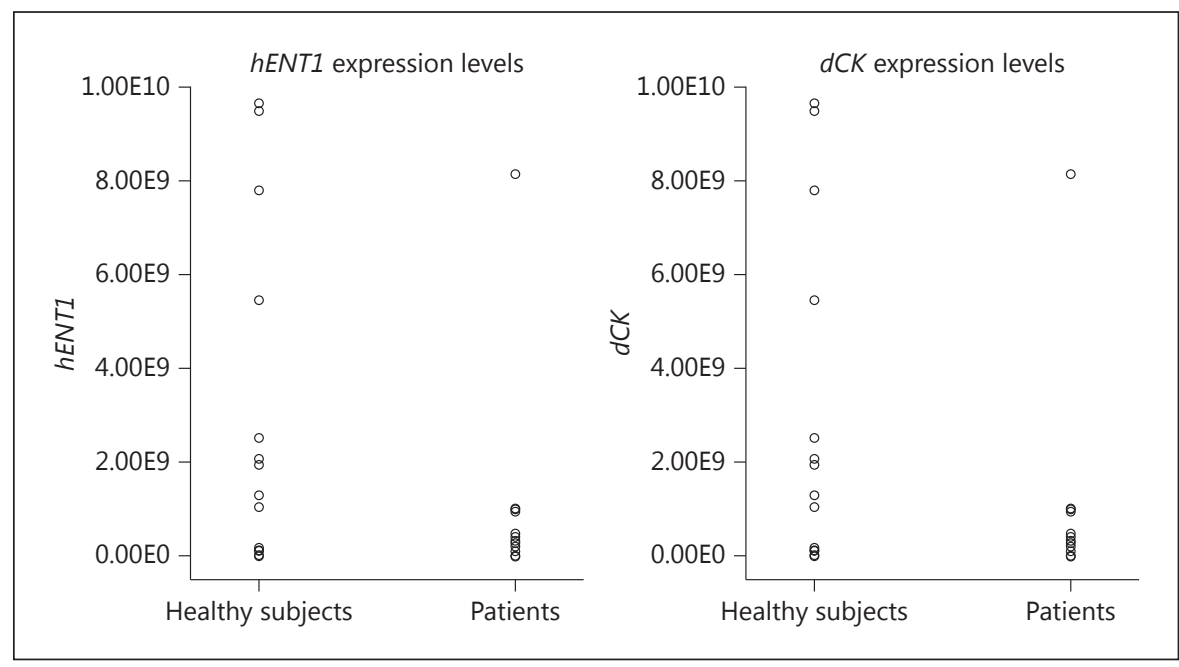

\section{Gene Expression Levels}

No blast selection was done to analyze gene expression but, in all patients, $>75 \%$ of circulating cells were blasts. Initially, expression levels of $h E N T 1$ and $d C K$ were graded in comparison with the expression of $18 S$. No correlation was found between $h E N T 1$ or $d C K$ expression levels and clinical baseline characteristics including cytogenetic risk, gender, age and morphological AML classification.

Expression levels of $h E N T 1$ and $d C K$ were compared with response rate, and arbitrarily 'normal levels' were defined as the median within responder patients. This cut-off level was compared with a nonresponder population. hENT1 expression levels were considered overexpressed or normal in 8 cases (29\%) and underexpressed in most cases $(\mathrm{n}=20,71 \%)$. In contrast, $d C K$ expression levels were evaluated as underexpressed in 13 cases (46.4\%) and considered normal or overexpressed in more cases $(53.6 \%)$.

Thereafter, expression levels of both genes were compared with a second, new cut-off obtained from 50 healthy volunteers. Of interest, we found that for $h E N T 1$ expression, the leukemic patients were within the range of the healthy volunteers but none could be considered as having underexpression of the $d C K$ gene. This comparison is shown in figure 1.

\section{Response}

Five patients died during the aplasia period. Therefore, response rates were evaluated in 23 patients. Complete response was achieved in 65\% of cases. Cox regression analysis determined that blasts percentage in bone marrow $(\mathrm{p}=0.01)$ and $h E N T 1$ and $d C K$ expression levels $(\mathrm{p}=0.012$ and $\mathrm{p}=0.04$, respectively) influenced patient
Table 2. Cox regression analysis to evaluate factors influencing treatment response

\begin{tabular}{|c|c|c|}
\hline \multicolumn{2}{|l|}{ Characteristics } & Significance \\
\hline \multicolumn{3}{|l|}{ Leukocytes } \\
\hline Range & $1,000-389,700$ & n.s. \\
\hline$<10,000 / \mathrm{mm}^{3}$ & 64.7 & \\
\hline$\geq 10,000 / \mathrm{mm}^{3}$ & 35.3 & \\
\hline \multicolumn{3}{|l|}{ Hemoglobin, g/dl } \\
\hline$<8$ & 23.5 & n.s. \\
\hline $8-10$ & 41.2 & \\
\hline$>10$ & 35.3 & \\
\hline \multicolumn{3}{|l|}{ Platelets, $/ \mathrm{mm}^{3}$} \\
\hline$<30,000$ & 29.4 & n.s. \\
\hline $30,000-50,000$ & 29.4 & \\
\hline $51,000-99,000$ & 11.8 & \\
\hline$\geq 100,000$ & 11.8 & \\
\hline \multicolumn{3}{|l|}{$\mathrm{LDH}$ value } \\
\hline Normal & 29.4 & n.s. \\
\hline Increased & 70.6 & \\
\hline \multicolumn{3}{|l|}{ Albumin, g/dl } \\
\hline$<2.5$ & 5.9 & n.s. \\
\hline $2.5-3.5$ & 58.8 & \\
\hline $3.6-4.6$ & 35.3 & \\
\hline \multicolumn{3}{|c|}{ Percentage of bone marrow blasts } \\
\hline $20-39$ & 11.8 & 0.012 \\
\hline $40-49$ & 17.6 & \\
\hline$>50$ & 70.6 & \\
\hline \multicolumn{3}{|c|}{$h E N T 1$ expression levels } \\
\hline Normal & 40 & 0.012 \\
\hline Underexpressed & 60 & \\
\hline \multicolumn{3}{|l|}{$d C K$ expression levels } \\
\hline Normal & 36 & 0.04 \\
\hline Underexpressed & 64 & \\
\hline
\end{tabular}

Values are expressed as percentages, unless otherwise indicated. $\mathrm{LDH}=$ Lactate dehydrogenase; n.s. = not significant. 


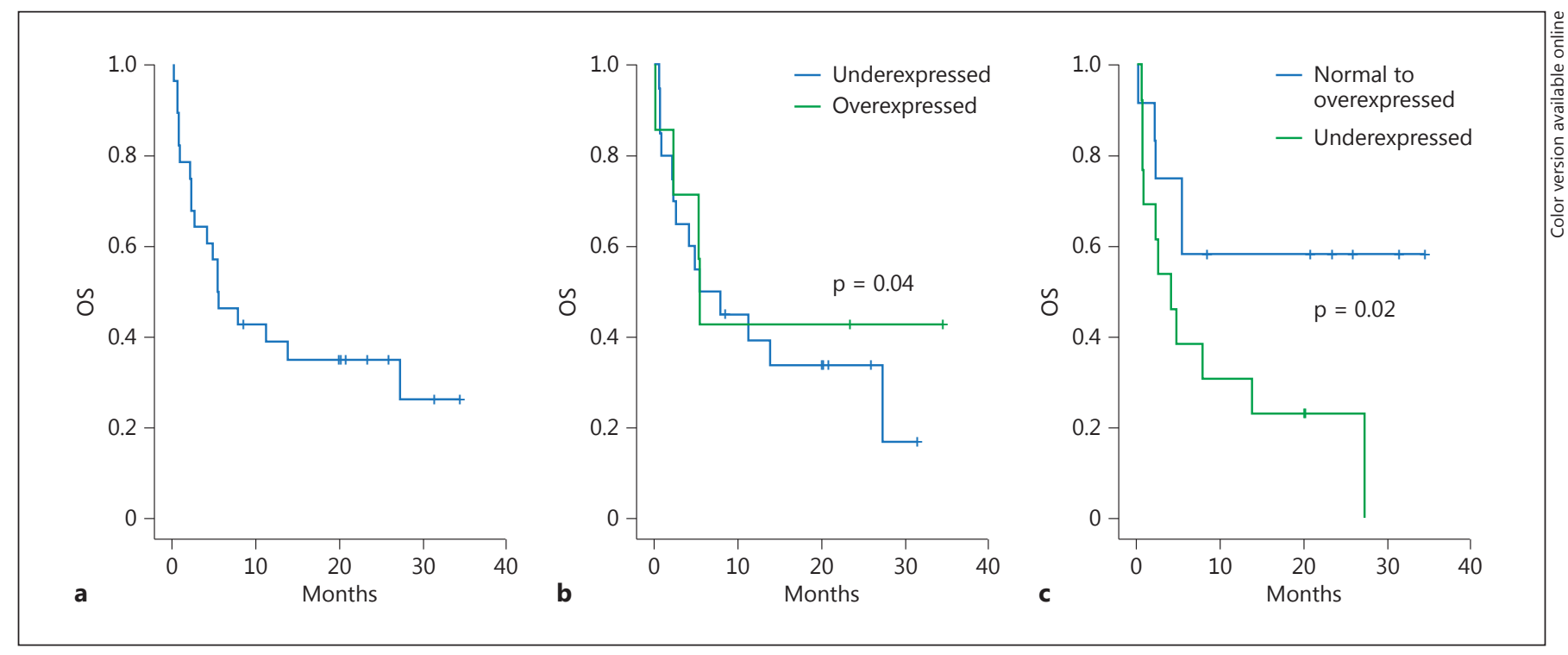

Fig. 2. a OS in the whole group. b OS according to $h E N T 1$ expression. c OS according to $d C K$ expression.

response (table 2). Patients with normal levels of $h E N T 1$ or $d C K$ enzyme genes had a higher response rate and survival (table 3 ).

\section{Survival}

OS in the entire group was $58 \%$ at 1 year but decreased to $38 \%$ at 30 months (fig. 2). Gene expression levels were statistically significant because patients with normally expressed $h E N T 1$ or $d C K$ had a $50 \%$ longer median OS (table 3$)$.

\section{Discussion}

Ara-C is included in different treatment schemas, and it remains the standard of care for AML patients. However, resistance to Ara-C incapacitates the therapeutic effort during treatment. Long-term results of standard treatment in adults with AML range from minimal to optimal. The health economic impact of induction chemotherapy using high-dose Ara-C compared to the standard dose was proposed by Fedele et al. [11]. High-dose Ara-C has a higher toxicity rate and higher costs for patients who require complete hospitalization until neutrophil recovery, and this schema is only adopted for young and fit patients [12]. Along the same lines, allotransplantation has been proposed for normal-karyotype AML patients who achieve a median survival of almost 18 months [13].
Table 3. Gene expression levels

\begin{tabular}{lrll}
\hline Levels & $\mathrm{n}$ & $\begin{array}{l}\text { Response } \\
\text { rate, \% }\end{array}$ & $\begin{array}{l}\text { Median } \\
\text { survival, } \\
\text { months }\end{array}$ \\
\hline hENT1 normal & 8 & 57 & $15.1^{*}$ \\
hENT1 underexpressed & 20 & 50 & $10.5^{*}$ \\
$d C K$ normal & 15 & 53.8 & 15.1 \\
$d C K$ underexpressed & 13 & 61.5 & 8.1 \\
\hline$*$ p $<0.05$. & & & \\
\hline
\end{tabular}

Nonbiological factors, including insurance status and county-level income, have also been associated with a poorer outcome [14], as in our series of patients in which we documented a $17.8 \%$ mortality rate during the aplasia period. Therefore, biomarkers related to predicted drug response are required in AML patients who are candidates for Ara-C treatment.

Ara-C is a prodrug that requires internalization into the cell via $h E N T 1$. Thereafter, the dCK enzyme produces a monophosphorylated product (dCMP), which is biand triphosphorylated to act as an antimetabolite. Resistance to Ara-C has been documented in AML cells with defective $d C K$ or $h E N T 1$ expression $[12,15,16]$. Our results clearly show that recently diagnosed, untreated AML patients with higher $h E N T 1$ and $d C K$ expression 
levels have a higher response rate and a better OS. Notably, higher expression levels of the $h E N T 1$ and $d C K$ genes correlated with better input in patients who were treated with other pyrimidine nucleotide analogs for pancreatic, lung and biliary tract cancer [17-20]. In particular, a recently published meta-analysis [17] concluded that pancreatic cancer patients with high $h E N T 1$ expression had longer disease-free survival with a hazard ratio of 2.62. These results are similar to in the study by Greenhalf et al. [18] in pancreatic patients who received adjuvant gemcitabine after resection.

The importance of $h E N T 1$ for Ara-C influx to cells was demonstrated previously, and this transporter defines the scope of new drugs or chemical modifications of Ara-C, such as the addition of elaidic acid to create the elaidic acid ester derivative of Ara-C or elacytarabine, a modified drug that has cellular influx independent of $h E N T 1$. Clinical trials to evaluate the efficacy of this drug are ongoing. Preliminary results [3] in AML patients with persistent blasts after the first induction course showed an encouraging overall response rate of $41 \%$ and an acceptable safety profile.

Other metabolic steps are also implicated in chemotherapy-resistant leukemic cells including kinase activities, such as inactivation of the dCK enzyme [21,22]. This modification was also documented in clinical conditions, including pancreatic, esophageal and biliary tract cancer $[23,24]$. Therefore, other analogs as clofarabine were evaluated in Ara-C-resistant leukemia [25]. Clofarabine primarily requires $h E N T 2$, and only a minor proportion of $h E N T 1$, to be transported into the cell, and it also requires phosphorylation by $\mathrm{dCK}$ and deoxyguanosine kinase. The sensitivity of Ara-C-resistant leukemia to clofarabine may be related to the affinity of this drug for other transporters and activation by other kinases [2628].

Advances in cancer genomics have revealed a spectrum of somatic mutations that give rise to human AML, drawing our attention to its molecular evolution and clonal architecture [26-28]. Therefore, new target drugs were developed and evaluated in AML patients either alone or in combination with chemotherapy [28]. As an example, the addition of cladribine to regimens containing Ara-C and anthracyclines (idarubicine) allows not only a higher complete remission rate, including patients with unfavorable karyotype, but also has a more significant advantage in OS [29]. However, the cost of these new regimens may be unacceptable for patients with limited resources. Therefore, the optimization of standard treatments requires evaluation using a pharmacogenetic and pharmacoeconomic scope.

\section{Disclosure Statement}

The authors declare that they have no conflict of interest.

\section{References}

1 Wiernick PH: Optimal therapy for adult patients with acute myeloid leukemia in first complete remission. Curr Treat Options Oncol 2014;15:171-186.

2 Candelaria M, Taja-Chayeb L, Arce-Salinas $\mathrm{C}$, et al: Genetic determinants of cancer drug efficacy and toxicity. Practical considerations and perspectives. Anticancer Drugs 2005;16: 923-933.

3 Rizzieri D, Vey N, Thomas X, Huguet-Rigal F, et al: A phase II study of elacytarabine in combination with idarubicin and of human equilibrative nucleoside transporter 1 expression in patients with acute myeloid leukemia and persistent blasts after the first induction course. Leuk Lymphoma 2014;55:2114-2119.

4 Chen E, Johnnson E: Characterization of the deoxycytidine kinase promoter in human lymphoblast cell lines. J Clin Invest 2005;95: 1660-1668.

5 Hatford CM, Duan S, Delaney SM, et al: Population-specific genetic variants important in susceptibility to cytarabine arabinoside cytotoxicity. Blood 2009;113:2145-2153.
6 Kroep JR, Loves WJP, van der Wilt Cl, et al: Pretreatment deoxycytidine kinase levels predict in vivo gemcitabine sensitivity. Mol Cancer Ther 2002;1:371-376.

7 Song JH, Kim SH, Kweon SH, et al: Defective expression of deoxycytidine kinase in cytarabine-resistant acute myeloid leukemia cells. Int J Oncol 2009;34:1165-1171.

8 Rathe S: Deoxycytidine kinase is downregulated in Ara-C-resistant acute myeloid leukemia murine cell lines. Leukemia 2010;24: 1513-1515.

9 Livak KJ, Schmittgen TD: Analysis of relative gene expression data using real time quantitative PCR and the 2(-Delta Delta C(T). Methods 2001;25:402-408.

10 Yalcin A: Quantification of thieredoxin mRNA expression in the rat hippocampus by real-time PCR following oxidative stress. Acta Biochim Pol 2004;51:1059-1065.
11 Fedele P, Avery S, Patil S, et al: Health economic impact of high-dose versus standarddose cytarabine induction chemotherapy for acute myeloid leukaemia. Intern Med J 2014; 44:757-763.

12 Mata JE, Scrideli Ca, Queiroz RP, et al: Cytosine arabinoside-metabolizing enzyme genes are underexpressed in children with MLL gene-rearranged acute lymphoblastic leukemia. Braz J Med Biol Res 2006;39:1417-1423.

13 Percival ME, Medeiros BC, Tian L: Allogeneic hematopoietic cell transplant for normal karyotype acute myeloid leukemia: indirect evidence of selection for adverse molecular profile. Bone Marrow Transplant 2015;50: 1004-1006.

14 Borate UM, Mineishi S, Costa LJ: Nonbiological factors affecting survival in younger patients with acute myeloid leukemia. Cancer 2015;121:3877-3884. 
15 Hummel-Eisenbeiss J, Hascher A, Hals PA, et al: The role of human equilibrative nucleoside transporter 1 of the cellular transport of the DNA methyltransferase inhibitors 5-azacytidine and CP-4200 in human leukemic cells. Mol Pharmacol 2013;84:438-450.

16 Yamauchi T, Negoro E, Kishi S, et al: Intracellular cytarabine triphosphate production correlates to deoxycytidine kinase/cytosolic $5^{\prime}$-nucleotidase II expression ratio in primary acute myeloid leukemia cells. Biochem Pharmacol 2009;77:1780-1786.

17 Zhu Y, Qi M, Lao L, et al: Human equilibrative nucleoside transporter 1 predicts survival in patients with pancreatic cancer treated with gemcitabine: a meta-analysis. Genet Test $\mathrm{Mol}$ Biomarkers 2014;18:306-312.

18 Greenhalf W, Ghaneth P, Neoptolemos JP, et al: Pancreatic cancer hENT1 expression and survival from gemcitabine in patients from the ESPAC-3 trial. J Natl Cancer Inst 2014; 106:djt347.

19 Poplin E, Wasan H, Rolfe L, et al: Randomized, multicenter, phase II study of CO-101 versus gemcitabine in patients with metastatic pancreatic ductal adenocarcinoma: including a prospective evaluation of the role of hENT1 in gemcitabine or CO-101 sensitivity. J Clin Oncol 2013;31:4453-4461.
20 Murata A, Amano R, Yamada N, et al: Prognostic predictive values of gemcitabine sensitivity-related gene products for unresectable or recurrent biliary tract cancer treated with gemcitabine alone. World J Surg Oncol 2013; 11:117.

21 Candelaria M, de la Cruz-Hernandez E, TajaChayeb L, et al: DNA methylation-independent reversion of gemcitabine resistance by hydralazine in cervical cancer cells. PLoS One 2012; 7:e29181.

22 Saiki Y, Yoshino Y, Fujimura H, et al: DCK is frequently inactivated in acquired gemcitabine-resistant human cancer cells. Biochem Biophys Res Commun 2012;421:98104.

23 Shimada Y, Okumura T, Sekine S, et al: Clinicopathological significance of deoxycytidine kinase expression in esophageal squamous cell carcinoma. Mol Clin Oncol 2013;1:716720 .
24 McAllister F, Pineda DM, Jimbo M, et al: dCK expression correlates with 5-fluorouracil efficacy and HuR cytolasmic expression in pancreatic cancer: a dual-institutional follow-up with the RTOG 9704 trial. Cancer Biol Ther 2014;15:68-98.

25 Yamauchi T, Uzui K, Nishi R, et al: Cytarabine-resistant leukemia cells are moderately sensitive to clofarabine in vitro. Anticancer Res 2014;34:1657-1662.

26 Grove CS, Vassiliou GS: Acute myeloid leukemia: a paradigm for the clonal evolution of cancer? Dis Model Mech 2014;7:941-951.

27 Larkin K, Blum W: Novel therapies in AML: reason for hope or just hype? Am Soc Clin Oncol Educ Book 2014:e341-e351.

28 Song G, Valdez BC, Li Y, Liu Y, et al: Synergistic cytotoxicity of sorafenib with busulfan and nucleoside analogs in human FLT3-ITDpositive acute myeloid leukemia cells. Biol Blood Marrow Transplant 2014;20:16871695.

29 Shen Y, Chen J, Wu D: Addition of cladribine to idarubicin and cytarabine during induction increases the overall efficacy rate in adult patients with acute myeloid leukemia: a matched-pair retrospective comparison. Chemotherapy 2014;60:368-374. 